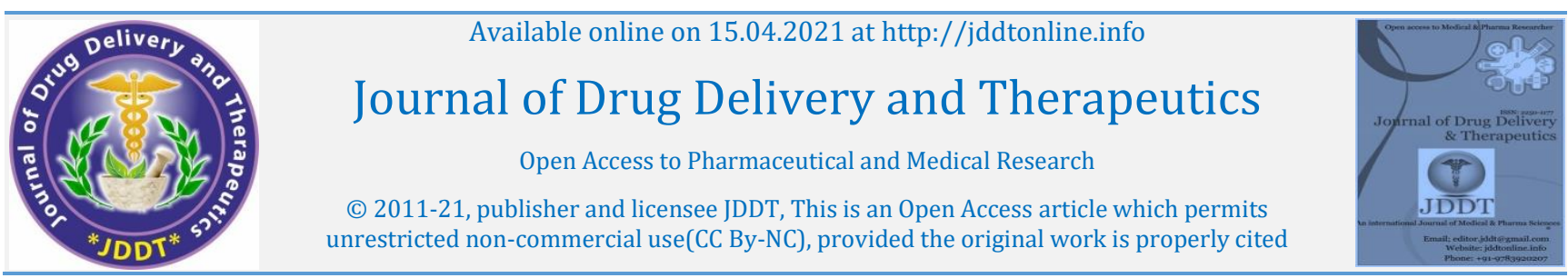

(C) 2011-21, publisher and licensee JDDT, This is an Open Access article which permits

Open Access Full Text Article

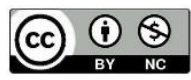

Research Paper

\title{
Development and In-Vitro Evaluation of Budesonide Mucoadhesive Microsphere for Pulmonary Drug Delivery
}

\author{
Jeevan Patel *, Revathi A Gupta \\ Institute of Pharmacy, Dr. A. P. J. Abdul Kalam University, Indore-Dewas bypass, Arandia, Indore - 452016 (M.P.)
}

\section{Article Info:}

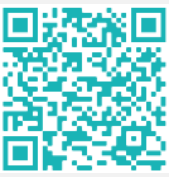

\section{Article History:}

Received 07 Feb 2021

Review Completed 20 March 2021

Accepted 28 March 2021

Available online 15 April 2021

\section{Cite this article as:}

Patel J, Gupta RA, Development and In-Vitro Evaluation of Budesonide Mucoadhesive Microsphere for Pulmonary Drug Delivery, Journal of Drug Delivery and Therapeutics. 2021; 11(2-s):76-81 DOI: http://dx.doi.org/10.22270/jddt.v11i2-s.4622

*Address for Correspondence:

Jeevan Patel, Institute of Pharmacy, Dr. A. P. J. Abdul Kalam University, Indore-Dewas bypass, Arandia, Indore - 452016 (M.P.)

\section{Abstract}

The purpose of the study was to develop and evaluate mucoadhesive microspheres of Budesonide for pulmonary drug delivery system having prolonged residence time and sustained drug release. Microspheres were prepared by emulsification solvent evaporation technique using HPMC, carbopol as polymers in varying ratios. The microspheres were evaluated for its percentage yield, drug entrapment efficiency, particle size and shape, in vitro mucoadhesion study and in vitro drug release studies. The FTIR studies revealed no chemical interaction between the drug molecule and polymers and found that drug was compatible with used polymer. The mucoadhesive microspheres showed particle size, drug entrapment efficiency and yield in the ranges of $148-164 \mu \mathrm{m}, 68.0-85.0 \%$ and $67.52-$ $87.25 \%$ respectively. In vitro drug release and mucoadhesion study confirms that formulation F5 was the best formulation as it releases $81.8 \%$ at the end of $12 \mathrm{hr}$. in controlled manner and percentage mucoadhesion of $75.2 \%$ after $10 \mathrm{hr}$. This confirms the developed budesonide mucoadhesive microspheres are promising for pulmonary drug delivery system.

Keywords: Budesonide, Mucoadhesion, Microspheres, Drug entrapment efficiency.

\section{INTRODUCTION}

Pulmonary drug delivery system has been used for the treatment of local and systemic diseases ${ }^{1}$. The local respiratory diseases like chronic obstructive pulmonary disease (COPD) and asthma were the prime focus in the early studies; however the research has been advanced in the treatment of respiratory infectious diseases and also in the transport of therapeutic molecules to the systemic circulation. Respiratory system in itself restricts the entrance of particulate matter by various means: e.g. geometry of the airways and clearance mechanisms of the lungs.The conventional inhalation products have high dosing frequency often leads to local and systemic toxicity. Therefore, novel controlled release carrier systems may provide a probable solution to these difficulties by reducing dosing frequency and increasing drug bioavailability2,3.

Drug action can be improved by developing new oral controlled drug delivery system, such as the mucoadhesive microsphere 4 . The concept of bioadhesion and specifically mucoadhesion is one of them to increase gastric retention time of drugs.Mucoadhesion or bioadhesion can be defined as the state in which two materials, at least one of which is biological in nature, are held together for a prolonged time period by means of interfacial forces.These systems remain in close contact with the absorption tissue, the mucous membrane, releasing the drug at the action site leading to anincrease bioavailability and both local and systemic effects ${ }^{5}$. The oral route of drug administration constitutes the most convenient and preferred means of drug delivery to systemic circulation of body. However oral administration ofmost of the drugs in conventional dosage forms hasshortterm limitations due to their inability torestrain and localize the system at gastro-intestinaltract.

Mucoadhesive microspheres helps to achieve relatively short residence time by the site of absorption can be overcome by coupling bioadhesion characters developed and to improve localization of oral controlled or sustained release drug delivery system by intimate contact with the mucous layer and by specific targeting of drug to the absorption site 6 . Microspheres of biodegradable or non-biodegradable polymers can be used for sustained delivery ${ }^{7}$.

Budesonide is a locally acting glucocorticoid with high topical anti-inflammatory activity. It has been used for many years in the treatment of inflammatory airway diseases for the low systemic activity of budesonide due to its rapid metabolism to biologically inactive. One of the most common uses of budesonide is in ulcerative colitis and Crohn's disease ${ }^{8}$.

It has a low bioavailability of $9-21 \%$, because of its poor absorption in gastro intestinal tract. It undergoes extensive, primarily hepatic metabolism after oral administration'. Therefore, in the present study budesonide is selected as a 
suitable drug for the design of mucoadhesive microspheres with a view to improve its oral bioavailability and increase its drug release in a sustained manner ${ }^{10}$.

\section{MATERIALS AND METHODS}

\section{Material}

Budesonide was obtained as gift- sample from Cipla Pharmaceutical Pvt. Ltd. Indore and carbopol 934, HPMC of pharmaceutical gradewere procured from Loba chemicals Pvt Ltd and liquid paraffin, Span 80, ethanol, acetone procured from SD Fine chemicals.

\section{Pre-formulation studies:}

Pre- formulation is considered as important phase where researcher characterizes the physical and chemical properties of drug substance which helps to develop stable, effective and safe dosage forms and also check possible interaction with various excipients ${ }^{8}$.

\section{Compatibility Study FT-IR Spectra Analysis}

FT-IR Spectroscopy can be used to investigate and predict any physicochemical interactions between different components in a formulation ${ }^{11}$. While selecting the ingredients, we would choose those which are stable, compatible and therapeutically acceptable.The aim of compatibility study was to test, whether there is any interaction between the excipients and the drug and compatibility between the drug and excipients. The pellets were prepared and scanned over a wave number range of $4000-500 \mathrm{~cm}^{-1}$ in a FTIR instrument.FT-IR spectrum of budesonide was compared with FT-IR spectra of budesonide with excipients. The puredrug and drug with excipients were scanned separately. Disappearance of budesonide peaks or shifting of peak in any of the spectra was studied ${ }^{12}$.

Preparation of Mucoadhesive microspheres by Emulsification-solvent evaporation

In the present study, mucoadhesive polymers carbopol 934, HPMC were used in different ratios with the active ingredient for the preparation of mucoadhesive microspheres. These polymers were employed for the fact that they possess good biocompatibility, non-irritant and non-toxic ${ }^{13}$. Accurately weighted amount of the polymers carbopol 934, HPMC as shown in Table No. 1 were dissolved in $50 \mathrm{ml}$ of acetone to form a homogenous polymers solution. Budesonide was then dispersed in it and mixed thoroughly. This dispersion quickly injected into $5 \mathrm{ml}$ liquid paraffin containing $1 \%(\mathrm{w} / \mathrm{w})$ of Span-80 by using a $5 \mathrm{ml}$ of syringe at room temperature with stirring at $1000 \mathrm{rpm}$ to form a uniform emulsion ${ }^{14,15}$. Thereafter, it was allowed to attain room temperature and stirring was continued until residual acetone evaporated and smooth-walled, rigid and discrete microspheres were formed. The microspheres were collected by decantation and the product was washed with n-hexane and stored in desiccators over fused calcium chloride ${ }^{16,17}$.

Table 1: Formulation design of Mucoadhesive microspheres

\begin{tabular}{|c|c|c|c|c|c|c|c|}
\hline \multirow{2}{*}{ S.No. } & \multirow{2}{*}{ Ingredients } & \multicolumn{7}{|c|}{ Formulation Code } \\
\cline { 3 - 8 } & & F1 & F2 & F3 & F4 & F5 & F6 \\
\hline 1 & Drug(mg) & 10 & 10 & 10 & 10 & 10 & 10 \\
\hline 2 & HPMC(mg) & 50 & 100 & 150 & 150 & 150 & 100 \\
\hline 3 & Carbopol 934(mg) & 50 & 100 & 150 & 50 & 100 & 150 \\
\hline 4 & Light Paraffin(ml) & 50 & 50 & 50 & 50 & 50 & 50 \\
\hline 5 & Span 80(ml) & 1 & 1 & 1 & 1 & 1 & 1 \\
\hline 6 & Acetone(ml) & 50 & 50 & 50 & 50 & 50 & 50 \\
\hline
\end{tabular}

\section{Evaluation of Prepared Mucoadhesive Microspheres:}

The mucoadhesive microspheres evaluation was carried out for appropriate assessment of a dispersed system requires characterization of both chemical and physical stabilities.Physical properties are very important with respect to the performance of dispersed systems ${ }^{18}$.

\section{Percentage yield}

The total amount of microspheres obtained werecalculated and evaluated for percentage yield 19 .

Percentage yield was calculated using the formula:

Percentage yield = Practical yield/Theoretical yield $x 100$

\section{Degree of Swelling}

The degree of swelling is a property measured to know the behaviour of polymer in physiological solution. It is determined by keeping the microspheres in buffer solution for $24 \mathrm{hrs}$ and washed. The swelling ability of mucoadhesive microspheres in physiological media was determined by swelling them in the PBS pH 6.8. Accurately weighed $20 \mathrm{mg}$ of microspheres were immersed in little excess of PBS pH 6.8 for $8 \mathrm{hrs}$ and washed ${ }^{19}$.

The degree of swelling was calculated using following formula,

$$
\alpha=(W s-W o) / W o
$$

Where, $\alpha$ is the degree of swelling

Wo is the weight of microspheres before swelling

Wsis the weight of microspheres after swelling.

\section{Particle Size Determination}

Determination of average particle size of Mucoadhesive microspheres loaded with Budesonide was carried out by using optical microscopy. A minute quantity of microspheres was spread on a clean glass slide and average size of 300 microspheres was determined in each batch.

\section{Scanning Electron Microscopy (SEM)}

The microspheres were observed under a Scanning Electron Microscopy. They were mounted directly onto SEM sample 
stub using double-sided sticking tape and coated with gold film with ion spillter with gold target with resolution $3 \mathrm{~nm}$ (30 KV HV Mode),10 nm (30 KV HV Mode), $40 \mathrm{~nm}$ (30 LV Mode) and a vacuum system is fitted to it 20 .

\section{Drug Loading and Entrapment Efficiency}

For determining drug loading and entrapment efficiency, weighed the quantity of microspheres were dissolved in methanol and kept during the night on a mechanical shaker to extract the complete entrapped drug. The obtained solution was filtered through Whatman filter paper and analyzed by using a UV spectrophotometer at $246 \mathrm{~nm}$. The drug loading (DL) and entrapment efficiency (EE) were determined as per equations mentioned below ${ }^{20}$.

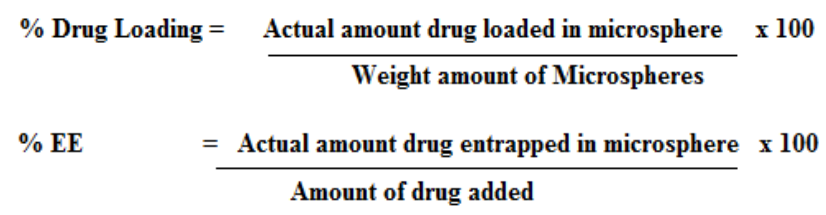

\section{In vitro Mucoadhesion Study (Wash off test)}

The in-vitro wash off test was carried out to evaluate the mucoadhesive potential of the microspheres.A small portion of the sheep intestinal mucosa was mounted on a glass slide and accurately weighed microspheres were sprinkled on the mucosa. Around 100 microspheres were spread on the wet mucosa and the prepared slide was hung onto one of the grooves of the USP tablet disintegrating test apparatus filled with Phosphate buffer solution $\mathrm{pH} 6.8$ giving regular up and down movements for 60 minutes. At the end of $60 \mathrm{~min}$, numbers of microspheres still adhering to the intestinal mucosa were counted ${ }^{20}$. The percentage of mucoadhesion was calculated by following formula:

\section{$\%$ Mucoadhesion $=\left(W a-W_{l}\right) \times 100 / W a$}

Where, $\mathbf{W a}=$ weight of microspheres applied

$\mathbf{W}_{\mathbf{l}}=$ weight of microspheres leached out.

\section{In vitro Drug Release Study}

In-vitro drug release study was carried out in USP dissolution test apparatus.Mucoadhesive microspheres were loaded into the basket of the dissolution apparatus. Dissolution study was carried out for $24 \mathrm{hrs}$ in media of phosphate bufferpH 6.8 at $37 \pm 0.5^{\circ} \mathrm{C}$ and at $100 \mathrm{rpm} .5 \mathrm{ml}$ of the sample was withdrawn from the dissolution media at suitable time intervals and diluted to $10 \mathrm{ml}$ using $\mathrm{pH} 6.8$ phosphate buffer and the same amount was replaced with fresh buffer.The absorbance was measured at $246 \mathrm{~nm}$ by using Shimadzu 1700 UV spectrophotometer, against a blank solution ${ }^{19,20}$.

\section{RESULT AND DISCUSSION}

\section{Compatibility Study FT-IR Spectra Analysis}

The comparative FTIR studies of Drug and excipients combination revealed that no chemical interaction between the drug molecule and polymers and found that drug was compatible with used polymer.The FTIR spectra of pure drug and drug with excipients are shown in the Fig 1, 2 \&3.

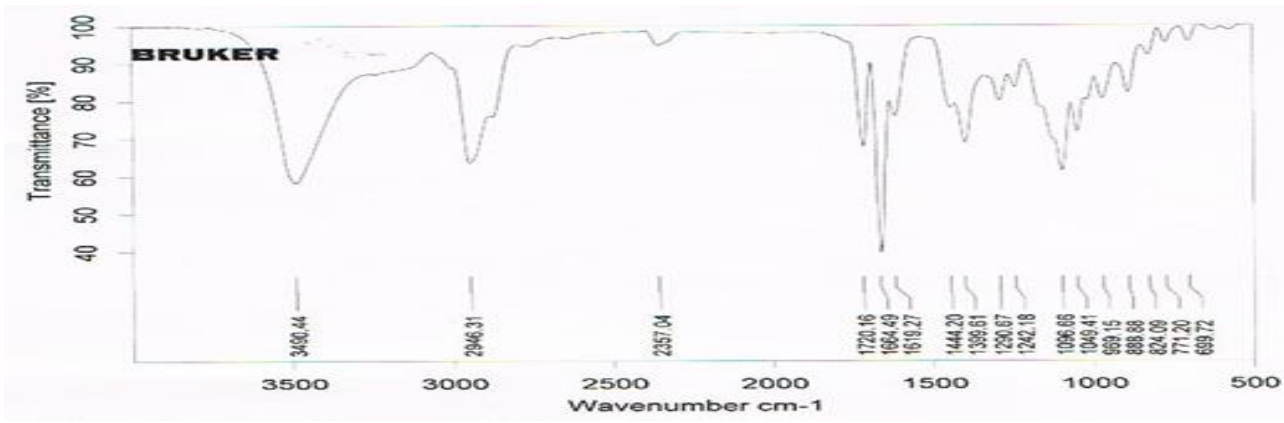

Figure 1: FT-IR Spectra of pure drug (BUDESONIDE)

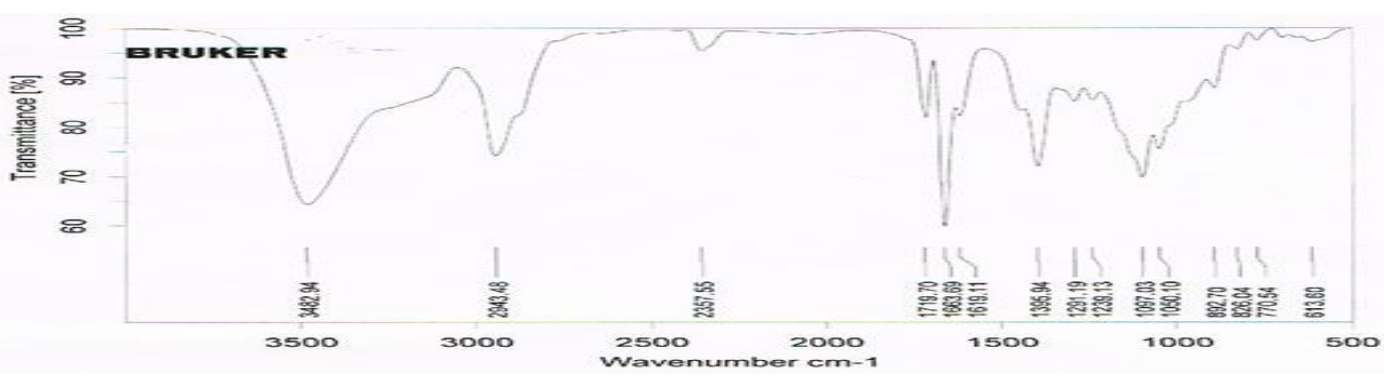

Figure 2: FT-IR Spectra of Budesonide with Polymer (HPMC)

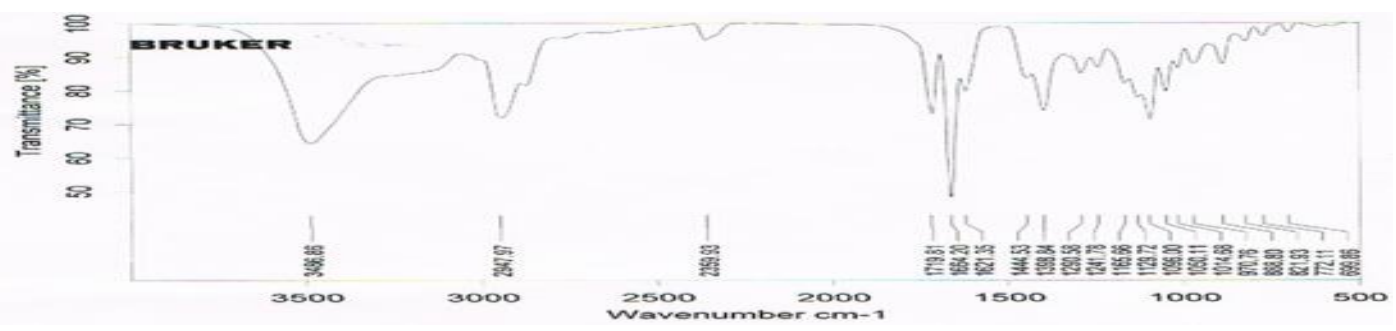

Figure 3: FT-IR Spectra of Budesonide with Polymer (Carbopol) 


\section{Percentage Yield}

The percentage yield of microspheres was calculated by using the weight of final product after drying with respect to initial total weight. The maximum percentage yield was found of F5 batch and was noted to be $87.25 \%$ among all the batches. The production yields of microspheres were found to be between 67.52 to $87.25 \%$ as shown in Table 2 . The percentage yields are shown in Fig 5.

\section{Degree of Swelling}

Degrees of swelling of the formulations were carried out and found to be within the range between 1.38 - 1.85 .

\section{Particle Size Determination}

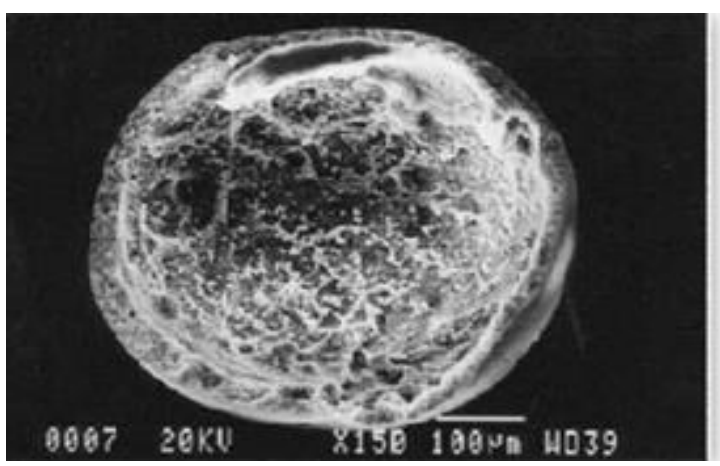

With increase in polymer concentration, the mean particle size of the microspheres significantly increased and range was between 148 to $164 \mu \mathrm{m}$. The particle sizes of Budesonide microspheres are shown in Fig 5.

\section{Scanning Electron Microscopy (SEM)}

The morphology of the mucoadhesive microspheres of best formulation $\mathrm{F} 5$ was examined by scanning electron microscopy. SEM confirms the outer surface of F5 formulation was smooth and dense, while the internal surface was porous. The shell of microspheres also showed some porous structure it may be caused by evaporation of solvent entrapped within the shell of microspheres after forming smooth and dense layer. The scanning electron microscopy of F5 batch is shown on Fig 4.

Figure 4: Scanning Electron Microscopy

\section{Drug Loading and Entrapment Efficiency}

Percent Entrapment Efficiency of the formulations was found to be within the range between 68.0 to $85.0 \%$. The maximum percentage yield was found of F5 batch and was noted to be $85.4 \%$ among all the batches. The percentage entrapment efficiency is shown in Table 2\& Fig 5.

\section{In vitro Mucoadhesion Study}

Prepared microspheres were found good mucoadhesion strength. Percent mucoadhesion of the all batches of microspheres were found to be in the range of 65.0 to
81.42\%.On increasing the polymer concentration, the bioadhesive property of the microspheres also increased as shown in Fig. 5. Increase in polymer concentration was attributed to increase in viscosity; produce stronger mucus gel network which helps to increase mucoadhesion ${ }^{21}$. The percentage mucoadhesion of microspheres adhering to tissue after $60 \mathrm{~min}$. is displayed in Table 2. The F5 batch showed highest \% mucoadhesion which was attributed to increase in concentration of polymers.

Table 2: Physicochemical Properties and Characterization of Budesonide Mucoadhesive Microspheres

\begin{tabular}{|c|c|c|c|c|c|}
\hline Formulation code & $\begin{array}{c}\text { Particle Size } \\
\text { (in } \boldsymbol{\mu m} \text { ) }\end{array}$ & $\begin{array}{c}\text { Percentage } \\
\text { yield (in \%) }\end{array}$ & $\begin{array}{c}\text { Entrapment } \\
\text { Efficiency (in \%) }\end{array}$ & $\begin{array}{c}\text { Degree of } \\
\text { swelling }\end{array}$ & $\begin{array}{c}\text { Percent mucoadhesion } \\
\text { (in \%) }\end{array}$ \\
\hline F1 & 148 & 75.82 & 75.2 & 1.82 & 65.0 \\
\hline F2 & 160 & 67.52 & 68.6 & 1.73 & 67.71 \\
\hline F3 & 156 & 76.64 & 80.3 & 1.38 & 75.71 \\
\hline F4 & 162 & 77.17 & 75.6 & 1.55 & 73.42 \\
\hline F5 & $\mathbf{1 6 4}$ & $\mathbf{8 7 . 2 5}$ & $\mathbf{8 5 . 4}$ & $\mathbf{1 . 8 5}$ & $\mathbf{8 1 . 4 2}$ \\
\hline F6 & 152 & 83.37 & 82.5 & 1.36 & 70.0 \\
\hline
\end{tabular}




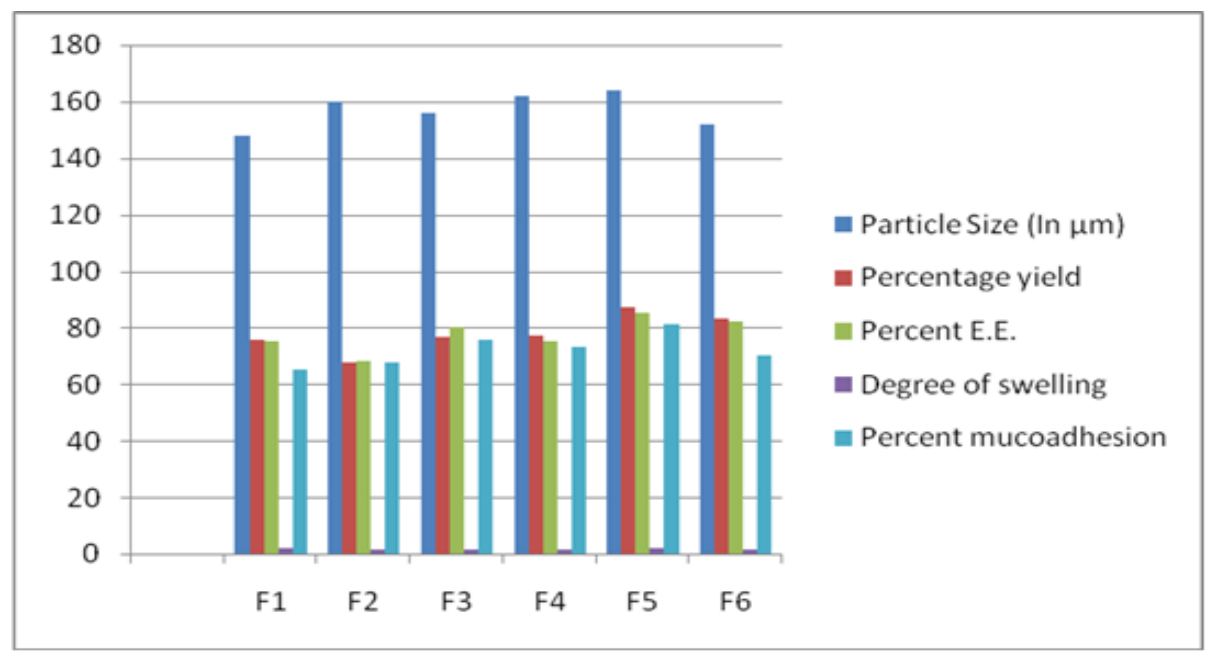

Figure 5: Graphical representation of Physicochemical Properties and Characterization of Budesonide Mucoadhesive Microspheres

\section{In vitro dissolution study}

The drug release from budesonide microsphere was carried out in pH 6.8 buffers as a dissolution medium for a period of 24 hrs respectively. The in-vitro budesonide release profile for all batches was shown in Fig. 6. Drug release from these mucoadhesive microspheres were slow, controlled release and dependent upon the nature and concentration of mucoadhesive polymers used 22,23 . It was found that there was decrease in budesonide release with increase in mucoadhesive polymer content. Hence it is considered as the best microsphere formulation which seems to be a good candidate for controlled release. The microspheres were subjected to in-vitro drug release rate by dissolution profiles are shown inTable 3 . In vitro drug release study confirms that the formulation F5 was the best formulation as it releases $81.8 \%$ at the end of $12 \mathrm{hr}$. in controlled manner

Table 3: In vitro release of Mucoadhesive microspheres in media

\begin{tabular}{|c|c|c|c|c|c|c|}
\hline Time(hr) & $\begin{array}{c}\text { \%CDR in } \\
\text { F1 }\end{array}$ & $\begin{array}{c}\text { \%CDR } \text { in } \\
\text { F2 }\end{array}$ & $\begin{array}{c}\text { \%CDR in } \\
\text { F3 }\end{array}$ & $\begin{array}{c}\text { \%CDR in } \\
\text { F4 }\end{array}$ & $\begin{array}{c}\text { \%CDR in } \\
\text { F5 }\end{array}$ & $\begin{array}{c}\text { \%CDR in } \\
\text { F6 }\end{array}$ \\
\hline $\mathbf{0}$ & 0 & 0 & 0 & 0 & 0 & 0 \\
\hline $\mathbf{0 . 5}$ & 10.7 & 8.2 & 9.6 & 9.1 & 10.2 & 10.3 \\
\hline $\mathbf{1 . 0}$ & 15.2 & 12.5 & 17.1 & 10.2 & 15.6 & 14.3 \\
\hline $\mathbf{2 . 0}$ & 25.5 & 23.2 & 26.3 & 20.2 & 24.6 & 21.6 \\
\hline $\mathbf{4 . 0}$ & 35.9 & 35.1 & 39 & 36.2 & 35.3 & 39.3 \\
\hline $\mathbf{6 . 0}$ & 44.3 & 46.3 & 44.3 & 48.3 & 48.1 & 52.6 \\
\hline $\mathbf{8 . 0}$ & 55.5 & 55.8 & 52.5 & 51.2 & 66.2 & 61.9 \\
\hline $\mathbf{1 0 . 0 0}$ & 64.82 & 73.3 & 72.2 & 74.7 & 75.2 & 72.3 \\
\hline $\mathbf{1 2 . 0 0}$ & 81.8 & 76 & 75.3 & 63.6 & 81.8 & 78.4 \\
\hline $\mathbf{1 8 . 0 0}$ & 85.3 & 80.2 & 78 & 72.3 & 87.7 & 81.3 \\
\hline $\mathbf{2 4 . 0 0}$ & 92.07 & 83.2 & 81 & 75.5 & & 93.2 \\
\hline
\end{tabular}

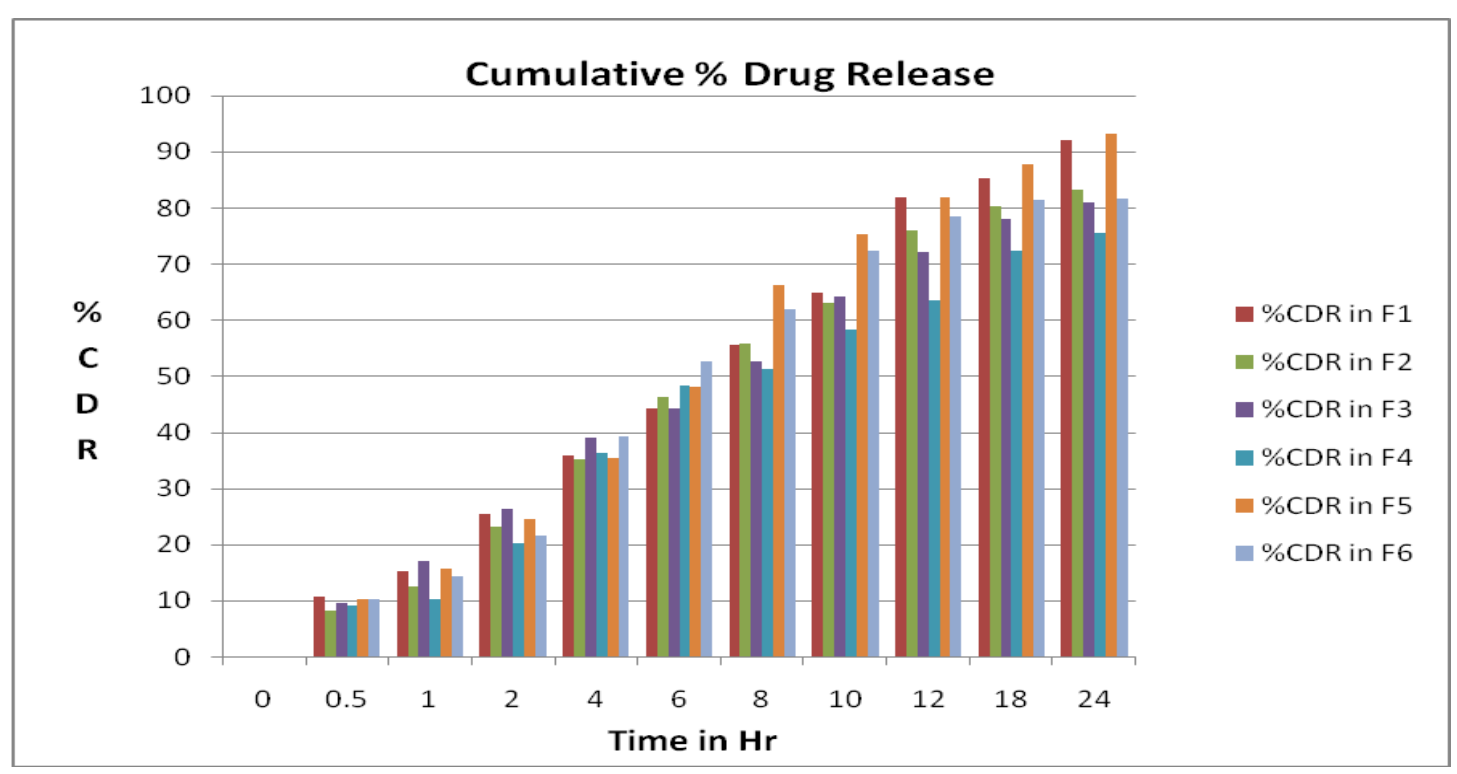

Figure 6: In vitro release of Mucoadhesive microspheres 


\section{CONCLUSION}

In the present study, a satisfactory attempt has been made to formulate mucoadhesive microspheres of budesonide for pulmonary drug delivery. Budesonide undergoes extensive hepatic metabolism. Hence mucoadhesive microspheres were developed to enhance the bioavailability and to prepare sustain microspheres having slow release in gastrointestinal tract. Microsphere formulation of budesonide was prepared by using emulsion solvent evaporation method. From the experimental study, it was concluded that in vitro drug release and mucoadhesion study confirms thatformulationF5 was the best formulation as it releases $81.8 \%$ at the end of $12 \mathrm{hr}$. in controlled manner and percentage mucoadhesion of $75.2 \%$ after $10 \mathrm{hr}$. In vitro release of mucoadhesive microspheres of budesonide was found to be in sustained manner and is dependent on the concentration of polymer used. It was found that there was decrease in budesonide release with increase in mucoadhesive polymer content and increased bioadhesive property of the microspheres with increasing the polymer concentration. Hence, it was concluded that the prepared mucoadhesive microspheres of budesonide prove to be potential system for safe and effective sustained release over an extended period of time for pulmonary drug delivery. In future the research can be extended to in-vivo studies to prove the increased bioavailability statistically.

\section{Acknowledgement}

The authors are thankful to Institute of Pharmacy, Dr. A. P. J. Abdul Kalam University, Indore - 452016 (Madhya Pradesh), India for their valuable support and permission to carry out the work.

\section{Conflict of Interest}

There is no conflict of interest.

\section{REFERENCES}

1. Carvalho FC, Bruschi ML, Evangelista RC, Gremi MPD. Mucoadhesive drug delivery system. Brazilian Journal of Pharmaceutical Sciences 2010; 46(1):1-17.

2. Parmar H, Bakliwal S, Gujarathi N, Rane B, Pawar S. Different method of formulation and evaluation of mucoadhesive microsphere. International Journal of Applied Biology and Pharmaceutical Technology 2010; 1(3):1157-1167.

3. Shaikh R, Singh TRR, Garland MJ, Donnelly RF.Mucoadhesive Drug Delivery Systems. Journal of Pharmacy and Bioallied Sciences 2011; 3(1): 89-100. Alexander A, Tripathi DK, Verma T, Patel S. Mechanism responsible for mucoadhesion of mucoadhesive drug delivery system. International Journal of Applied Biology and Pharmaceutical Technology 2011; 2(1):434-445.

4. Punitha S, Girish Y. Polymers in mucoadhesive buccal drug delivery system. International Journal of Research and Pharmaceutical Sciences 2010; 1(2):170-186.

5. Ganga S. Mucosal Drug Delivery. Pharmainfo.net 2007; 5.
6. Sachan NK, Bhattacharya A. Basic and Therapeutic Potential of Oral Mucoadhesive Microparticulate Drug Delivery Systems. International Journal of Pharmaceutical and Clinical Research 2009; 1:10.

7. Bharathi A, Mandava H, Sekhar N, Chandra D, Ramakrishna V, Formulation and Evaluation of Budesonide Controlled-ileal Release Pellets Int J Pharma Res Health Sci. 2018; 6(4):2685-89

8. Kala D and Nair CRS: Formulation and In vitro Evaluation of Budesonide Microspheres for Colon Targeting. Int J Pharm Sci Res 2017; 7(2):697-02. doi: 10.13040/IJPSR.09758232.7(2).697-02.

9. Kala D, Sudhakaran Nair CR: Formulation and In vitro Evaluation of Budesonide Microspheres for Colon Targeting. Int J Pharm Sci Res 2016; 7(2):697-02.doi: 10.13040/IJPSR.0975-8232.7(2).697-02.

10. Hemlata Kaurav, S. L. HariKumar and Amanpreet Kaur Mucoadhesive Microspheres as carriers in Drug Delivery: a Review / International Journal of Drug Development \& Research April-June 2012; 4(2).

11. Nanomedicine in pulmonary delivery Heidi M Mansour YunSeok Rhee Xiao Wu University of Kentucky, College of Pharmacy, Division of Pharmaceutical Sciences-Drug Development Division,

12. Tomoko Oosegi, Hiraku Onishi, Yoshiharu Machida. Novel preparation of enteric-coated chitosan-prednisolone conjugate microspheres and in vitro evaluation of their potential as a colonic delivery system. Euro J Pharm Biopharm. 2008; 68:260-6.

13. Haznedar. B, Dortun. B. Preparation and in vitro evaluation of Eudragit microspheres containing acetazolamide. Int J Pharm. 2004; 269:131-40.

14. Yunying Tao, Yifan Lu, Yinjing Sun, Bing Gu, Weiyue Lu, Jun Pan. Development of mucoadhesive microspheres of acyclovir with enhanced bioavailability. Int J Pharm.2009; 378:30-6.

15. Martinac. A, Filipovi'c-Gřcic. J, Voinovich. D, Perissutti. B, Franceschinis. E, Development and bioadhesive properties of chitosan-ethylcellulose microspheres for nasal delivery. Int Pharm. 2005; 291:69-77.

16. Arya RKK, Singh R, Vijay Juyal. Mucoadhesive microspheres of famotidine: preparation Characterization and in vitro Evaluation. Int J Eng Sci Tech. 2010; 2(6):1575-80.

17. Yellanki SK, Singh J, Syed JA, Rajkamal Bigala, Sharada Goranti, Nerella NK. Design and Characterization of Amoxicillin trihydrate Mucoadhesive Microspheres for Prolonged Gastric retention. Int J Pharm Sci and Drug Res 2010; 2(2):112-114.

18. Hardenia SS, Jain A, Patel R, Anu Kaushal. Formulation and Evaluation of Mucoadhesive Microspheres of Ciprofloxacin. J Adv Pharm Edu Res. 2011; 1(4):214-24.

19. Sathiya Sundar R, Murugesan A, Venkatesan P, Manavalan R Formulation development and evaluation of Carprofen Microspheres. 2010; 2(3):1674-76.

20. Patel JK, Patel RP, Amin AF, Patel MM. Formulation and evaluation of mucoadhesive glipizide microspheres AAPS pharm Sci Tech 2004 Oct 12. avalable from URL: http://www.aapspharmscitech.org.

21. Prajapati SK, tripati P, Ubaidulla U, Anand V. Design and Development of Gliclazide Mucoadhesive Microcapsules: in vitro and in vivo Evaluation. AAPS Pharm Sci Tech. 2008 March; 9(1).available from URL:http://www.aapspharmsciech.org.

22. Gavin PA, Laverty TP, Jones DS. Mucoadhesive Polymeric Platforms for Controlled Drug Delivery. European Journal of Pharmaceutics and Biopharmaceutics 2009; 71:505-518. 